\title{
RESILIÊNCIA DE PESSOAS COM DIABETES MELLITUS ${ }^{1}$
}

\author{
Bárbara Cristina Tavares², Francine do Amaral Barreto ${ }^{3}$, Marília Lima Lodetti ${ }^{4}$, Denise Maria Guerreiro \\ Vieira da Silva ${ }^{5}$ Juliana Cristina Lessmann ${ }^{6}$
}

${ }^{1}$ Artigo originado do Trabalho de Conclusão de Curso - Resiliência de pessoas com Diabetes Mellitus, apresentado ao Curso de Graduação em Enfermagem da Universidade Federal de Santa Catarina (UFSC), 2009.

${ }^{2}$ Enfermeira do Hospital Universitário da UFSC. Santa Catarina, Brasil. E-mail: barbaratavares22@gmail.com

${ }^{3}$ Enfermeira. Santa Catarina, Brasil. E-mail: fran_barreto@hotmail.com

${ }^{4}$ Enfermeira. Santa Catarina, Brasil. E-mail: ma.lima87@gmail.com

${ }^{5}$ Doutora em Enfermagem. Professora Associado do Departamento de Enfermagem e do Programa de Pós-Graduação em Enfermagem (PEN) da UFSC. Bolsista de produtividade do CNPq. Santa Catarina, Brasil. E-mail: denise@ccs.ufsc.br

${ }^{6}$ Doutoranda em Enfermagem PEN/UFSC. Bolsista CAPES/REUNI. Santa Catarina, Brasil E-mail: julianalessmann@gmail.com

RESUMO: O estudo teve como objetivo caracterizar a resiliência e variáveis sócio-demográficas e de saúde/doença de pessoas com Diabetes Mellitus atendidas em um hospital geral de Florianópolis, Santa Catarina, Brasil. Trata-se de um estudo transversal, desenvolvido com 60 sujeitos no período de agosto a novembro de 2009, utilizando a Escala de Resiliência de Connor-Davidson. Os escores de resiliência foram elevados, havendo associação entre resiliência e idade. Pessoas atendidas na emergência obtiveram escores de resiliência significativamente superior às pessoas do ambulatório e unidades de internação. Concluímos que pessoas com Diabetes Mellitus podem apresentar escores elevados de resiliência, compatível com uma população saudável e de países mais desenvolvidos. DESCRITORES: Diabetes Mellitus. Resiliência psicológica. Doença crônica. Enfermagem.

\section{RESILIENCE AMONG PEOPLE WITH DIABETES MELLITUS}

\begin{abstract}
This study aimed to characterize the resilience, socio-demographic, and health/disease variables among people with Diabetes Mellitus who were attended in a general hospital in Florianopolis, Santa Catarina, Brazil. This cross-sectional study was developed with 60 subjects from August to November of 2009, using the Connor-Davidson Resilience Scale. Resilience scores were high, with no association between age and resilience. People attended through emergency services obtained significantly superior resilience scores to those from outpatient and inpatient units. We conclude that people with diabetes may have high resilience scores, compatible with a healthy population and more developed countries.
\end{abstract}

DESCRIPTORS: Diabetes Mellitus. Resilience psychological. Chronic disease. Nursing.

\section{RESILIENCIA DE LAS PERSONAS CON DIABETES MELLITUS}

RESUMEN: El estudio tuvo por objetivo investigar la resiliencia y las variables socio-demográficas y de salud/enfermedad de las personas con Diabetes Mellitus que acuden a un hospital general en Florianópolis, Santa Catarina, Brasil. Se trata de un estudio transversal, desarrollado con sesenta personas, en el periodo de agosto a noviembre de 2009, por medio de la Escala de Resiliencia Connor-Davidson. Los valores de resilencia fueron elevados, con una asociación entre resilencia y edad. Las personas atendidas en el servicio de urgencia presentaron valores de resilencia superiores al de las personas del ambulatorio y las unidades de internación. Llegamos a la conclusión que las personas con Diabetes Mellitus pueden tener puntuaciones más altas de resiliencia, compatibles con una población sana y de los países más desarrollados.

DESCRIPTORES: Diabetes Mellitus. Resiliencia psicológica. Enfermedad crónica. Enfermería. 


\section{INTRODUÇÃO}

O Diabetes Mellitus (DM) é um conjunto de alterações metabólicas caracterizadas por hiperglicemia crônica, em decorrência da destruição das células beta do pâncreas, resistência à ação e/ou distúrbios da secreção da insulina. ${ }^{1}$

O DM é uma doença cujos sinais e sintomas evoluem lentamente, dificultando a descoberta e o estabelecimento de diagnóstico precoce. Assim, muitas pessoas comumente recebem o diagnóstico após a manifestação de complicações da doença, como as cardiovasculares. ${ }^{2}$ Estimou-se que no Brasil, no ano de 2007, existiam 5,7\% de mulheres e $4,8 \%$ de homens que se reconheciam portadores do $\mathrm{DM}^{3}$

Essa doença crônica não transmissível requer adaptações ao estilo de vida e incorporação de práticas terapêuticas que envolvem alterações de padrões alimentares, realização de controle glicêmico, prática de atividades físicas, manutenção da pressão arterial, e acompanhamento contínuo da equipe multidisciplinar de saúde. ${ }^{2}$

Viver com o DM implica em ajustar-se à complexa dinâmica entre as relações familiares, sentimentos, estilo de vida e as mudanças de hábitos, adequação de rotinas, implementação de cuidados e procedimentos de controle da glicemia, objetivando um viver saudável com o DM e a prevenção das complicações agudas e crônicas. ${ }^{4}$

A participação da equipe de saúde no processo de cuidado de pessoas com DM envolve a prevenção de complicações, a promoção da saúde e o estabelecimento de ações de educação para o fortalecimento da adesão aos tratamentos medicamentosos e comportamentais. ${ }^{4-5}$

Desta forma, o enfermeiro necessita estabelecer vínculo com a pessoa, promovendo a troca de informações e permitindo que ela expresse seus sentimentos em relação a sua situação de saúde-doença, auxiliando na adesão ao tratamento e nas mudanças no estilo de vida. ${ }^{6}$

As percepções acerca de uma doença nunca são as mesmas para as diferentes pessoas, ocorrendo manifestações e reações singulares em cada uma delas. ${ }^{7}$ Algumas conseguem superar os desafios e manter a doença sob controle, tendo uma vida saudável e harmoniosa. No entanto, outras encaram a doença como um grande problema, não conseguindo ter uma vida plena. ${ }^{7}$

Desta forma, a maneira como as pessoas percebem sua condição influencia no controle geral do seu estado de saúde-doença, ${ }^{8-9}$ sendo que o enfoque da resiliência traz a possibilidade de novos olhares acerca do viver com o DM.

O termo resiliência está relacionado aos processos psicossociais que beneficiam o desenvolvimento do ser humano, auxiliando-o no convívio com as adversidades e problemas enfrentados ao longo de sua vida. ${ }^{8,10} \mathrm{O}$ termo resiliência é comumente assumido como uma reafirmação da capacidade humana em superar adversidades e situações de risco como o estresse e experiências sociais adversas. ${ }^{12-13}$

A noção de resiliência tem origem na física, sendo associada à capacidade máxima de um determinado material de suportar tensão sem se deformar de maneira permanente. ${ }^{11} \mathrm{Na}$ área da saúde alguns estudos vêm sendo realizados, porém, não há estudos específicos envolvendo resiliência e diabetes, conforme levantamento efetuado nas bases Medline/PubMed, Lilacs, CINAHL, Cochrane, Scopus, Isi Web of Science e Scielo.

Acredita-se que as situações de "risco e proteção" são peças fundamentais para o desenvolvimento da resiliência. ${ }^{13}$ Os fatores de proteção podem ser a autoestima, o apoio social e a aproximação interpessoal, e estão aliados à capacidade de enfrentar os fatores de risco e mudar o curso da vida da pessoa, para uma evolução favorável. ${ }^{13}$

A resiliência também pode ser compreendida como a capacidade individual de lidar com a doença, aceitando suas limitações, colaborando com aderência ao tratamento, readaptando-se e sobrevivendo de forma positiva ${ }^{8}$, podendo ser desenvolvida e ampliada ao longo da vida. ${ }^{14}$

Desse modo, o objetivo deste estudo foi caracterizar a resiliência e as variáveis sóciodemográficas e de saúde/doença de pessoas com DM atendidas em um hospital geral de Florianópolis-SC, Brasil.

\section{MÉTODO}

Trata-se de um estudo transversal, realizado em um hospital geral de Florianópolis-SC. A amostra intencional foi constituída de 60 pessoas com DM tipo 1 e 2 nas unidades de emergência adulto, ambulatório e clínicas de internação médica e cirúrgica, sendo 20 participantes em cada local.

Os critérios de inclusão dos sujeitos no estudo foram: ter recebido o diagnóstico de DM há mais de um ano e ter idade superior a 18 anos. Os critérios de exclusão adotados foram: não ter condições físicas ou emocionais de responder ao 
questionário; apresentar dificuldade de comunicação e/ou possuir déficit cognitivo.

A coleta de dados ocorreu entre de agosto e novembro de 2009, sendo realizada por três das pesquisadoras que, no momento da coleta, eram acadêmicas de enfermagem realizando o Trabalho de Conclusão de Curso. Para a seleção dos participantes da pesquisa eram realizadas visitas diárias aos locais do estudo e efetuado levantamento junto aos registros de enfermagem acerca das pessoas com DM internadas ou que teriam consultas de enfermagem no ambulatório. As pessoas com DM identificadas e que atendiam aos critérios de inclusão/exclusão eram convidadas a participar. As entrevistas tiveram duração média de 40 minutos.

Foram coletados dados sóciodemográficos (sexo, idade, religião, estado marital, escolaridade, pessoas com que residem no mesmo domicílio, renda individual e familiar, fonte de renda), além de dados relacionados ao DM (tempo que a pessoas conhece o diagnóstico do DM, mudanças na vida relacionadas ao DM, controle glicêmico, realização de tratamento medicamentoso e não medicamentoso, presença de complicações do DM e percepção acerca do estado de saúde). Estes dados integravam um instrumento desenvolvido pelas autoras. Também foi aplicada a Escala de Resiliência de Connor-Davidson - CD-RISC, ${ }^{15}$ traduzida por Trentini e Silva*.

O instrumento CD-RISC é composto de 25 itens, que medem competência pessoal, tolerância de afeto negativo e de apoio social. Os escores variam de 0 a 100, sendo que quanto maior pontuação, maior resiliência. ${ }^{15}$

Os dados coletados foram inseridos em um arquivo no programa Excel $^{\circledR}$ da Microsoft $^{\circledR} \mathrm{e}$ exportados para ferramenta computacional on-line SEstatNet/UFSC, ${ }^{16}$ sendo efetuada estatística descritiva de todas as variáveis. Um dos testes de hipóteses efetuado foi entre as variáveis 'escore geral de resiliência' e 'local de atendimento', sendo variável quantitativa discreta e qualitativa nominal, respectivamente. Como o modelo Normal não caracterizou a distribuição da variável 'escore geral de resiliência' em pelo menos uma das categorias da variável 'local de atendimento', o teste estatístico mais adequado foi o ANOVA de Kruskal-Wallis.
Para avaliar a associação entre 'escore geral de resiliência' e a variável 'escolaridade', os dados relativos à escolaridade foram subdivididos em duas categorias: analfabetos/ensino fundamental incompleto e ensino fundamental completo ou mais, sendo classificada como qualitativa ordinal. Como os escores de resiliência apresentaram normalidade e homocedasticidade nas duas categorias da variável escolaridade, foi empregado o teste $t$ de Student não pareado, com variância agregada.

Na avaliação da associação 'escore geral de resiliência' e a variável 'idade', também foi efetuada a categorização dos dados da variável idade em menor/igual a 60 anos e mais de 60 anos, sendo classificada como qualitativa ordinal. Como os escores de resiliência apresentaram normalidade e homocedasticidade nas duas categorias da variável idade, foi empregado o teste $t$ de Student não pareado com variância agregada.

A pesquisa seguiu os aspectos éticos previstos na Resolução n 196/96 do Conselho Nacional de Saúde ${ }^{17}$, respeitou o sigilo e o anonimato, aplicou o termo de consentimento livre e esclarecido, bem como foi aprovada sem restrições pelo Comitê de Ética da Universidade Federal de Santa Catarina, através do Processo n 221/09 (FR-276788), em julho de 2009.

\section{RESULTADOS}

Das 60 pessoas com DM investigadas, $60 \%$ eram mulheres e $40 \%$ homens, com média de idade de 77 anos, com desvio padrão (DP) de 12.6 anos, sendo a idade mínima de 19 anos, e a máxima, de 81 anos. A totalidade dos sujeitos seguia alguma religião, sendo a católica a mais frequente. A maioria tinha relação conjugal estável e residia com outras pessoas. Quanto à escolaridade, quase $70 \%$ cursaram até quatro anos de estudos, equivalente ao ensino fundamental incompleto. No que se refere à renda mensal, 70\% das pessoas com DM recebem até dois salários mínimos e cerca de $80 \%$ das famílias recebem até três salários mínimos. A fonte de renda, para $53 \%$, é a aposentadoria, sendo que o principal responsável pelo sustento da família era a própria pessoa com DM (Tabela 1).

\footnotetext{
* Tradução da escala de Connor e Davidson (2003) realizada por Trentini e Silva (2009) junto ao projeto de pesquisa "Mulheres com Diabetes Mellitus tipo 2: estresse e resiliência", que recebeu apoio financeiro por meio do edital MCT/CNPq/SPM-PR/MDA n ${ }^{\circ}$ 57/2008.
} 
Tavares BC, Barreto FA, Lodetti ML, Silva DMGV, Lessmann JC

Tabela 1 - Características sóciodemográficas de pessoas com Diabetes Mellitus. Florianópolis-SC, 2009

\begin{tabular}{|c|c|c|c|c|c|c|c|c|}
\hline \multirow{2}{*}{ Características sóciodemográficas } & \multicolumn{2}{|c|}{ Geral } & \multicolumn{2}{|c|}{ Emergência } & \multicolumn{2}{|c|}{$\begin{array}{c}\text { Clínica médica } \\
\text { e cirúrgica }\end{array}$} & \multicolumn{2}{|c|}{ Ambulatório } \\
\hline & $\mathbf{n}$ & $\%$ & $\mathbf{n}$ & $\%$ & $n$ & $\%$ & $\mathbf{n}$ & $\%$ \\
\hline \multicolumn{9}{|l|}{ Religião } \\
\hline Católica & 43 & 71,66 & 14 & 23,33 & 14 & 23,33 & 15 & 25,00 \\
\hline Evangélica & 11 & 18,34 & 3 & 5,00 & 4 & 6,67 & 4 & 6,67 \\
\hline Outras & 6 & 10,00 & 3 & 5,00 & 2 & 3,33 & 1 & 1,67 \\
\hline \multicolumn{9}{|l|}{ Estado marital } \\
\hline Solteiro & 4 & 6,67 & 1 & 1,67 & 3 & 5,00 & - & - \\
\hline Casado & 41 & 68,32 & 14 & 23,33 & 11 & 18,33 & 16 & 26,66 \\
\hline Viúvo & 10 & 16,67 & 4 & 6,67 & 6 & 10 & - & - \\
\hline Divorciado & 5 & 8,34 & 1 & 1,67 & - & - & 4 & 6,67 \\
\hline \multicolumn{9}{|l|}{ Com quem reside } \\
\hline Sozinho & 8 & 13,34 & 3 & 5,00 & 4 & 6,67 & 1 & 1,67 \\
\hline Pai/mãe & 1 & 1,67 & 1 & 1,67 & - & - & - & - \\
\hline Cônjuge & 14 & 23,33 & 3 & 5,00 & 3 & 5,00 & 8 & 13,33 \\
\hline Filhos (as) & 8 & 13,34 & 3 & 5,00 & 4 & 6,67 & 1 & 1,67 \\
\hline Cônjuge e filhos (as) & 26 & 43,32 & 10 & 16,66 & 7 & 11,66 & 9 & 15,00 \\
\hline Outros & 3 & 5,00 & - & - & 2 & 3,33 & 1 & 1,67 \\
\hline \multicolumn{9}{|l|}{ Escolaridade } \\
\hline Analfabeto; sabe ler ou escrever & 9 & 15,00 & 3 & 5,00 & 5 & 8,33 & 1 & 1,67 \\
\hline Ensino fundamental incompleto & 32 & 53,33 & 8 & 13,33 & 11 & 18,33 & 13 & 21,67 \\
\hline Ensino fundamental completo ou mais & 19 & 31,67 & 9 & 15,00 & 4 & 6,67 & 6 & 10,00 \\
\hline \multicolumn{9}{|l|}{ Renda individual } \\
\hline$\leq 2$ salários mínimos & 42 & 70,00 & 12 & 20,00 & 17 & 28,33 & 13 & 21,67 \\
\hline$\geq 3$ salários mínimos & 12 & 20,00 & 6 & 10,00 & 2 & 3,33 & 4 & 6,67 \\
\hline Não soube/Não respondeu & 6 & 10,00 & 2 & 3,33 & 1 & 1,67 & 3 & 5,00 \\
\hline \multicolumn{9}{|l|}{ Renda familiar } \\
\hline$\leq 2$ salários mínimos & 24 & 40,00 & 5 & 8,33 & 10 & 16,66 & 9 & 15,00 \\
\hline$\geq 3$ salários mínimos & 24 & 40,00 & 11 & 18,33 & 4 & 6,67 & 9 & 15,00 \\
\hline Não soube/Não respondeu & 12 & 20,00 & 4 & 6,67 & 6 & 10,00 & 2 & 3,33 \\
\hline \multicolumn{9}{|l|}{ Idade } \\
\hline$\leq 60$ anos & 25 & 41,66 & 5 & 8,33 & 6 & 10,00 & 14 & 23,33 \\
\hline$>60$ anos & 35 & 58,33 & 15 & 25,00 & 14 & 23,33 & 6 & 10 \\
\hline
\end{tabular}

Quanto ao tempo do diagnóstico da doença, $35 \%$ das pessoas sabiam que possuíam DM há mais de 10 anos, $31,67 \%$ possuíam o DM de seis a 10 anos, e 33,33\%, até cinco anos. A maioria respondeu que a doença modificou a sua vida em relação à rotina diária, a sensibilidade emocional, a restrição na alimentação e a utilização de medicamentos. Em relação ao tratamento, 91,67\% referiram que sempre seguem o tratamento medicamentoso; $5 \%$ às vezes; e 3,33\% referiram que não seguem. Ao investigar o objetivo do tratamento, 38,33\% referiram que é para a melhoria da qualidade de vida, 25,00\% para controlar o DM, $15, \%$ por medo de complicações e $21,67 \%$ não responderam.

Em relação à autoavaliação do controle glicêmico, 48,33\% referiram que mantém a glicemia controlada, porém $30,00 \%$ avaliaram que seu controle glicêmico sofre variações importantes, e somente às vezes está com bom controle. Para 21,67\% 
das pessoas, os valores da glicemia estão sempre alterados, denotando mau controle glicêmico.

No que se refere às complicações relacionadas ao DM, 62\% das pessoas com DM referiram possuir complicações decorrentes do DM, sendo as alterações visuais as mais frequentes. Estas complicações foram referidas como capazes de alterar as suas atividades diárias e o relacionamento com familiares e amigos.

Em relação à percepção de sua saúde, 51,67\% das pessoas com DM avaliaram como regular, $26,67 \%$ avaliaram como ruim e $21,66 \%$ consideraram-se em bom estado de saúde. Quanto à resiliência, a média geral foi de 77,96 , com desvio padrão de 12,56, ocorrendo variação expressiva nos escores, com mínimo de 46 e máximo de 100 (Tabela 2).

Tabela 2 - Resiliência de pessoas com Diabetes Mellitus, segundo local de atendimento e sexo. Florianópolis-SC, 2009

\begin{tabular}{lccccc}
\hline & \multicolumn{5}{c}{ Resiliência } \\
\cline { 2 - 6 } & Emergência & Clínica médica e cirúrgica & Ambulatório & Mulheres & Homens \\
\hline Média & 84,35 & 77,60 & 71,95 & 78,58 & 77,04 \\
Desvio-padrão & 7,67 & 13,41 & 13,03 & 12,61 & 12,68 \\
Mínimo & 72,00 & 53,00 & 46,00 & 46,00 & 52,00 \\
Máximo & 97,00 & 100,00 & 97,00 & 100,00 & 97,00 \\
10. Quartil & 79,50 & 69,00 & 60,50 & 69,50 & 71,00 \\
3o. Quartil & 90,50 & 87,50 & 80,00 & 88,00 & 86,00 \\
\hline
\end{tabular}

Ao analisar o escore de geral resiliência e o local de atendimento, por meio do teste ANOVA de Kruskal-Wallis, obteve-se um p-valor de 0,0061, mostrando que houve associação entre as duas variáveis. O teste de $t$ de Student mostrou que não há associação entre as variáveis escolaridade (analfabetos/ensino fundamental incompleto e ensino fundamental completo ou mais) e resiliência (p-valor de 0,2396$)$ e que há associação entre idade (menor/igual a 60 anos e mais de 60 anos) e resiliência (t de Student $=-2,7758$, p-valor=0,0037).

Ao subdividir a amostra segundo a idade das pessoas com DM e escores de resiliência, obteve-se que para aqueles com idade menor ou igual a 60 anos e com 60 anos ou mais a média de resiliência foi de 72,92 (DP 13,97) e 81,57 (DP 10,18), respectivamente.

\section{DISCUSSÃO}

O escore médio de resiliência obtido $(77,96)$ junto às pessoas com DM, que buscavam atendimento em um hospital (emergência, internação e ambulatório) foi semelhante ao encontrado no estudo de validação da escala, ${ }^{15}$ efetuado com uma população em geral $(80,4)$.

A justificativa relacionada a este achado está centrada na possibilidade de ocorrência de eventos negativos que permitirem uma reorganização da vida e vontade de seguir em frente ${ }^{13}$. Fato que reforça esta possibilidade foi a observação de que a maioria das pessoas entrevistadas apresenta DM há mais de cinco anos. Com base neste mesmo argumento, podemos acrescentar que as pessoas reconheceram que o DM modificou suas vidas, tanto em relação ao cotidiano quanto nas questões emocionais.

Analisando os escores entre os três locais onde as pessoas foram entrevistadas, percebemos que houve uma diferença significativa entre estas variáveis (p-valor de 0,0061 ), sendo que os escores das pessoas atendidas na emergência que foram bem mais elevados, quando comparados com as pessoas atendidas no ambulatório. Uma das possíveis justificativas para este achado é que a resiliência se desenvolve a partir do binômio fator de risco versus fator de proteção. ${ }^{11,13}$ Assim, pessoas que vivenciaram o agravamento de sua condição crônica e necessitaram de atendimento em uma unidade de emergência mobilizaram maior resiliência para o enfrentamento desta condição.

A comparação entre os escores médios da resiliência de homens e mulheres não foi significativa, resultado semelhante ao obtido em outros estudos, ${ }^{13,15}$ apesar de um desses estudos ter usado outro instrumento para a avaliação da resiliência.

Outra característica que pode ter contribuído para a melhor resiliência das pessoas estudadas é que a maioria referiu morar com outras pessoas, 
contando com apoio para conviverem melhor com a sua doença e superar as adversidades da vida. ${ }^{18}$ Um estudo ${ }^{18}$ também destaca que a baixa renda não é condição para baixa resiliência, fato que pode contribuir para a compreensão da similaridade entre os escores de resiliência das pessoas com DM entrevistas e os obtidos em uma população americana saudável. ${ }^{15}$

A alta taxa de adesão ao tratamento observada $(91,67 \%)$ diferiu do obtido em um estudo que apontou a taxa de até $50 \%$ para pessoas com DM. ${ }^{19}$ Acreditamos que este achado foi influenciado pela forma de coleta do dado, cuja pergunta foi realizada de forma genérica, sem avaliar o grau de efetividade e o nível de adesão.

Não houve diferença estatística significante entre resiliência e escolaridade, visto que o p-valor foi superior a 0,05. Já a relação entre resiliência e idade (menor/igual a 60 anos e mais de 60 anos) obteve significância, permitindo afirmar que pessoas com mais de 60 anos tem mais resiliência. Isso pode ser atribuído ao fato de que ao atingir a velhice já foram enfrentados vários acontecimentos adversos que ameaçam o processo de viver. ${ }^{20}$ Dessa forma, pessoas com maior idade podem ter maior experiência, o que pode contribuir para a aceitação dos limites da vida e provavelmente desenvolver o sentimento de pertencer a uma história mais ampla, mantendo o senso de integridade e ampliando sua resiliência. ${ }^{20}$

\section{CONCLUSÕES}

O acometimento por uma doença crônica, em especial do DM, supõe alterações significativas na vida de uma pessoa, não somente pelos efeitos deletérios da própria doença, mas também pelas medidas de controle glicêmico, dieta, realização de atividade física e possíveis mudanças no estado emocional.

Neste construto entendemos que o conceito de resiliência pode ser associado às pessoas com DM atendidas em unidades de emergência, unidade de internação e ambulatório. Verificamos que mesmo sendo portadores de uma doença crônica que pode causar complicações em sua saúde e de apresentarem idade avançada, baixa renda e baixa escolaridade, estas pessoas apresentaram escores elevados de resiliência, compatível com uma população saudável de um país desenvolvido. Assim, fatores de risco também podem elevar o nível de resiliência em pessoas com DM e não apenas os fatores de proteção estão associados a escores elevados. Convém lembrar que a amostra deste estudo foi intencional e que os achados não podem ser generalizáveis, porém apontam para a maior compreensão do DM e suas repercussões na vida e saúde das pessoas.

A compreensão de que a resiliência é dinâmica e pode ser desenvolvida reforça sua relevância na adaptação às demandas de saúde/doença e na melhoria da qualidade de vida de pessoas com DM, o que propõe novas perspectivas para o cuidado em saúde e enfermagem.

Recomenda-se que o enfoque da resiliência no cuidado de pessoas com DM seja reforçado, buscando o desenvolvimento de aspectos resiliêntes como a autoestima, a felicidade, o otimismo, a esperança, a satisfação e o fortalecimento das relações sociais e familiares para o apoio ao cuidado.

\section{REFERÊNCIAS}

1. American Diabetes Association. Standards of medical care in diabetes-2008. Diabetes Care [online]. 2008 [acesso 2010 Jun 15]; 31(1):S12-54. Disponível em: http:/ / care.diabetesjournals.org/ content/31/Supplement_1/S12.full.pdf+html

2. Sociedade Brasileira de Diabetes. Diretrizes da Sociedade Brasileira de Diabetes. Rio de Janeiro (RJ): SBD; 2008.

3. Ministério da Saúde (BR). Secretaria de Vigilância a Saúde. Secretaria de Gestão e Estratégia Participativa. Vigitel Brasil 2007: vigilância de fatores de risco e proteção para doenças crônicas por inquérito telefônico. Brasília (DF): Ministério da Saúde; 2009.

4. Silva DGV, Souza SS, Francioni FF, Matosinho MMS, Coelho MS, Sandoval RCB, et al. Pessoas com Diabetes Mellitus: suas escolhas de cuidados e tratamentos. Rev Bras Enferm. 2006 Maio-Jun; 59(3):297-302.

5. Cramer JA. A systematic review of adherence with medicathions for diaberes. Diabetes Care [online]. 2004 [acesso 2010 Ago 25]; 27(5):1218-24. Disponível em: http:/ / care.diabetesjournals.org/ content $/ 27 / 5 / 1218$.full.pdf + html?sid=b4105915fb38-4e1f-b06d-74b3f71cbf04

6. Xavier ATF, Bittar DB, Ataide MBC. Crenças no autocuidado em diabetes: implicações para a prática. Texto Contexto Enferm [online]. 2009 [acesso 2010 Jun 01]; 18(1):124-30. Disponível em: http:/ / www. scielo.br/pdf/tce/v18n1/v18n1a15.pdf

7. Camon, VAA. E a psicologia entrou no hospital. São Paulo (SP): Pioneira Thomson Learning; 2003.

8. Bianchini DCS, Dell'Aglio DD. Processos de resiliência no contexto de hospitalização: um estudo de caso. Paidéia [online]. 2006 [acesso 2010 Ago 10]; 16(35):427-36. Disponível em: http:/ / www.scielo. br/pdf/paideia/v16n35/v16n35a13.pdf 
9. Tavares DMS, Rodrigues FR, Silva CGC, Miranzi SSC. Caracterização de idosos diabéticos atendidos na atenção secundária. Ciênc Saúde Coletiva [online]. 2007 [acesso 2010 Jun 10]; 12(5):1341-52. Disponível em: http:/ / www.scielosp.org/pdf/csc/ v12n5/26.pdf

10. Silva MRS, Elsen I, Lacharite C. Resiliência: concepções, fatores associados e problemas relativos à construção do conhecimento na área. Paidéia [online]. 2003 [acesso 2010 Jun 10]; 13(26):14756. Disponível em: http://www.scielo.br/pdf/ paideia/v13n26/03.pdf

11. Pinheiro DPN. A resiliência em discussão. Psicol Estud [online]. 2004 [acesso 2010 Jun 23];9(1): 6775. Disponível em: http:/ / www.scielo.br/pdf/pe/ v9n1/v9n1a09.pdf

12. Morais NA, Koller SH. Abordagem ecológica do desenvolvimento humano, psicologia positiva e resiliência: ênfase na saúde. In: Koller SH, organizadora. Ecologia do desenvolvimento humano: pesquisas e intervenção no Brasil. São Paulo (SP): Casa do Psicólogo; 2004. p.91-107.

13. Pesce RP, Assis SG, Santos N, Oliveira RVC. Risco e proteção: em busca de um equilíbrio promotor de resiliência. Psic Teor Pesq [online]. 2004 [acesso 2010 Jun 23]; 20(2):135-43. Disponível em: http:/ /www. scielo.br/pdf/ptp/v20n2/a06v20n2.pdf

14. Taboada NG, Legal EJ, Machado N. Resiliência: em busca de um conceito. Rev Bras Crescimento Desenvolv Hum [online]. 2006 [acesso 2010 Jun 13]; 16(3):104-13. Disponível em: http:/ / pepsic.bvsalud. org/pdf/rbcdh/v16n3/12.pdf

15. Connor KM, Davidson JRT. Development of a new resilience scale: the Connor-Davidson Resilience
Scale (CD-RISC). Depress Anxiety [online]. 2003 [acesso 2010 Jun 13]; 18(2):76-82. Disponível em: http:// onlinelibrary.wiley.com/doi/10.1002/ da.10113/pdf

16. Nassar SM, Wronscki VR, Ohira M. SEstatNet - Sistema Especialista para o Ensino de Estatística na Web [página na internet]. Florianópolis (SC); UFSC; 2010 [atualizado 2010 Set 09; acesso 2009 Jun 15 a 2009 Dez 15]. Disponível em: http:/ /www.sestatnet.ufsc.br

17. Ministério da Saúde (BR), Conselho Nacional de Saúde, Comissão Nacional de Ética em Pesquisa. Resolução No 196 de 10 de outubro de 1996: diretrizes e normas regulamentadoras de pesquisa envolvendo seres humanos. Diário Oficial da União [online] 1996 Out 10 [acesso 2010 Abr 10]. Disponível em: http://conselho.saude.gov.br/resolucoes/ reso $96 . \mathrm{htm}$

18. Yunes MAM. Psicologia e resiliência: o foco no indivíduo e na família. Psicol Estud [online]. 2003 [acesso 2010 Jun 13]; 8(Esp):75-84. Disponível em: http:// www.scielo.br/pdf/pe/v8nspe/v8nesa10.pdf

19. Assunção TS, Ursine PGS. Estudo de fatores associados à adesão ao tratamento não farmacológico em portadores de Diabetes mellitus assistidos pelo Programa de Saúde da Família, Ventosa, Belo Horizonte. Ciênc Saúde Coletiva. 2008; 13(Suppl 2):2189-97.

20. Silva AI, Alves VP. Envelhecimento: resiliência e espiritualidade. - História de vida de idosos: superar as adversidades sem perder o senso de integridade. Diálogos Possíveis [online]. 2007 [acesso 2010 Jun 14]; 6(1):189-209. Disponível em: http:// www.faculdadesocial.edu.br/dialogospossiveis/ artigos/10/14.pdf
Correspondência: Denise Maria Guerreiro Vieira da Silva Universidade Federal de Santa Catarina Programa de Pós-Graduação em Enfermagem. Centro de Ciências da Saúde 88040-900 - Trindade, Florianópolis, SC, Brasil E-mail: denise@ccs.ufsc.br
Recebido: 17 de setembro de 2010 Aprovação: 4 de outubro de 2011 